\title{
Structural basis of the ultrasensitive calcium indicator GCaMP6
}

\author{
DING JingJin ${ }^{1,2 \dagger}$, LUO Andrew F. ${ }^{2 \dagger}$, HU LiYan ${ }^{2}$, WANG DaCheng ${ }^{1 *} \&$ SHAO Feng ${ }^{2 *}$ \\ ${ }^{1}$ National Laboratory of Biomacromolecules, Institute of Biophysics, Chinese Academy of Sciences, Beijing 100101, China; \\ ${ }^{2}$ National Institute of Biological Sciences, Beijing 102206, China
}

Received October 24, 2013; accepted November 11, 2013; published online January 2, 2014

\begin{abstract}
GCaMP is one of the most widely used calcium indicators in neuronal imaging and calcium cell biology. The newly developed GCaMP6 shows superior brightness and ultrasensitivity to calcium concentration change. In this study, we determined crystal structures of $\mathrm{Ca}^{2+}$-bound GCaMP6 monomer and dimer and presented detailed structural analyses in comparison with its parent version GCaMP5G. Our analyses reveal the structural basis for the outperformance of this newly developed $\mathrm{Ca}^{2+}$ indicator. Three substitution mutations and the resulting changes of local structure and interaction explain the ultrasensitivity and increased fluorescence intensity common to all three versions of GCaMP6. Each particular substitution in the three GCaMP6 is also structurally consistent with their differential sensitivity and intensity, maximizing the potential of using GCaMP6 in solving diverse problems in neuronal research and calcium signaling. Our studies shall also be beneficial to further structure-guided optimization of GCaMP and facilitate the design of novel calcium indicators.
\end{abstract}

GCaMP6, calcium indicator, structural basis, ultrasensitivity

Citation: Ding JJ, Luo AF, Hu LY, Wang DC, Shao F. Structural basis of the ultrasensitive calcium indicator GCaMP6. Sci China Life Sci, 2014, 57: 269-274, doi: $10.1007 / \mathrm{s} 11427-013-4599-5$

The intracellular concentration of free calcium ions $\left(\mathrm{Ca}^{2+}\right)$ changes quickly in excitable cells such as neurons and muscle cells [1,2]. Monitoring $\mathrm{Ca}^{2+}$ dynamics using specialized $\mathrm{Ca}^{2+}$ indicators could track the activity of neuronal populations and also excitation of a single neuron in real time [3-6]. Two types of $\mathrm{Ca}^{2+}$ indicators have been developed for $\mathrm{Ca}^{2+}$ imaging. The chemically synthesized $\mathrm{Ca}^{2+}$ indicators have high sensitivity and fast kinetics but could not be targeted to specific cells [7,8]; genetically encoded calcium indicators (GECIs) (also called fluorescent calcium indicator proteins; FCIPs) can enable long-term noninvasive imaging of defined cells and subcellular compartments but suffer from low brightness and slow response speed $[9,10]$. Extensive efforts have been made to develop effective $\mathrm{Ca}^{2+}$ indicators with desired performances in both cell targeting

$\dagger$ Contributed equally to this work

*Corresponding author (email: dcwang@ibp.ac.cn; shaofeng@nibs.ac.cn) and $\mathrm{Ca}^{2+}$ sensing.

GCaMP is one of the most widely used GECIs. GCaMP is developed by fusing a circularly permuted variant of enhanced green fluorescent protein (cpEGFP) with the calcium-binding protein calmodulin $(\mathrm{CaM})$ at the $\mathrm{C}$ terminus and a CaM-binding M13 peptide (from myosin light chain) at the $\mathrm{N}$ terminus [11]. Crystal structures of GCaMP2 reveal the structural basis for $\mathrm{Ca}^{2+}$ sensing by GCaMP $[12,13]$. In the absence of $\mathrm{Ca}^{2+}$, the chromophore in EGFP exists in the solvent-exposed neutral state as a result of EGFP inversion. Upon $\mathrm{Ca}^{2+}$ binding, the CaM moiety of GCaMP interacts with the M13 peptide, and the resulting CaM-M13 complex undergoes a significant structural reorganization, in proximity to the chromophore of cpEGFP. $\mathrm{Ca}^{2+}$-induced $\mathrm{CaM}$ and cpEGFP interaction blocks solvent access and restores the deprotonation of the chromophore, giving rising to a desired spectroscopic transition and measurable fluorescence changes $[12,13]$. GCaMP is a powerful tool for tracking 
$\mathrm{Ca}^{2+}$ dynamics in target cells, but has not yet acquired satisfactory sensitivity and kinetics comparable to chemically synthesized $\mathrm{Ca}^{2+}$ indicators. Structure-guided optimization has been performed to improve the fluorescence properties of GCaMP $[14,15]$. The most recently developed version GCaMP6 possesses long-sought ultrasensitivity to $\mathrm{Ca}^{2+} \mathrm{dy}$ namics [16]. GCaMP6 consists of three types, GCaMP6s, $6 \mathrm{~m}$, $6 \mathrm{f}$ (for slow, medium and fast kinetics, respectively). All the three types outperform their parent version GCaMP5G [15] in fluorescence intensity and signal-tonoise ratio, and their sensitivity to $\mathrm{Ca}^{2+}$ concentration change is comparable to that of the chemically synthesized $\mathrm{Ca}^{2+}$ indicator. Of all known GECIs, GCaMP6f shows the fastest response kinetics [16]. Among the three GCaMP6 versions, GCaMP6m has the most balanced performance between signal intensity and sensitivity. Its fluorescence intensity is as high as the highest version GCaMP6s, yet its response kinetics is faster [16].

To understand the structural basis underlying the superior property of GCaMP6, we determine crystal structures of $\mathrm{Ca}^{2+}$-bound GCaMP6m monomer and dimer at 2.7 and $2.49 \AA$, respectively. Comprehensive structural analyses provide mechanistic insights into how the improved fluorescence intensity and response speed of GCaMP6 are generated from substitution mutations of the parent version GCaMP5G.

\section{Materials and methods}

\subsection{Cloning, expression, and purification}

GCaMP6m with N-terminal RSET truncation was subcloned into a modified pET-28a(+) vector with a $\mathrm{His}_{6}$ tag, a SUMO tag, and a Ulp1 protease site at the $\mathrm{N}$ terminus to facilitate protein folding and purification. The recombinant plasmid was transformed into Escherichia coli strain BL21 (DE3) (Novagen, Germany). Proteins were expressed for $18 \mathrm{~h}$ at $295 \mathrm{~K}$ after induction with $0.5 \mathrm{mmol} \mathrm{L}^{-1}$ isopropylb-D-thiogalactoside. Cell pellets expressing recombinant GCaMP6m were harvested and lysed by sonication in lysis buffer $\left(20 \mathrm{mmol} \mathrm{L}^{-1}\right.$ HEPES $\mathrm{pH}$ 7.5, $0.2 \mathrm{~mol} \mathrm{~L}^{-1}$ sodium chloride and $10 \mathrm{mmol} \mathrm{L}^{-1}$ imidazole). After centrifugation, the soluble proteins were first purified by using Ni-NTA agarose beads (GE Healthcare, USA). The His ${ }_{6}$ and SUMO tags of STK25ct were removed by Ulp1 protease digestion. Subsequently, the monomeric and dimeric GCaMP6m were separated by size-exclusion chromatography using a Hiload 16/60 Superdex 200 column (GE Healthcare). The purified monomeric and dimeric proteins were concentrated to 10 $\mathrm{mg} \mathrm{mL} \mathrm{m}^{-1}$, and stored in a buffer containing $20 \mathrm{mmol} \mathrm{L}^{-1}$ HEPES pH 7.5, $150 \mathrm{mmol} \mathrm{L}^{-1} \mathrm{NaCl}$, and $1 \mathrm{mmol} \mathrm{L}^{-1} \mathrm{CaCl}_{2}$ at $193 \mathrm{~K}$.

\subsection{Crystallization and data collection}

Both GCaMP6m monomer and dimer crystals were grown by the hanging-drop vapor diffusion method at $293 \mathrm{~K}$ with $2 \mu \mathrm{L}$ drops containing $1 \mu \mathrm{L}$ protein solution and $1 \mu \mathrm{L}$ reservoir solution equilibrated over $500 \mu \mathrm{L}$ reservoir solution. GCaMP6m monomer crystals were obtained with reservoir buffer containing $0.1 \mathrm{~mol} \mathrm{~L}^{-1}$ HEPES $\mathrm{pH} 7.4,18 \% \mathrm{w} / \mathrm{v}$ polyethylene glycol 3350 . The crystals were soaked in a cryoprotectant solution containing 0.1 mol L $\mathrm{L}^{-1}$ HEPES $\mathrm{pH} 7.4,18 \% \mathrm{w} / \mathrm{v}$ polyethylene glycol 3350 , and $20 \%$ dimethyl sulfoxide before being flash-frozen with liquid nitrogen. X-ray diffraction data were collected on a Rigaku FR-E diffraction system using a Rigaku R-Axis IV++ image plate detector. GCaMP6m dimer crystals were obtained with reservoir buffer containing $0.1 \mathrm{~mol} \mathrm{~L}^{-1}$ HEPES pH 8.0, $20 \%$ w/v polyethylene glycol 3350 . The crystals were transferred through a solution containing $0.1 \mathrm{~mol} \mathrm{~L}^{-1} \mathrm{HEPES} \mathrm{pH} 8.0$, $20 \% \mathrm{w} / \mathrm{v}$ polyethylene glycol 3350 and $15 \%$ ethylene glycol before being flash-frozen with liquid nitrogen. X-ray diffraction data were collected on a Rigaku 007 diffraction system using a Rigaku Saturn CCD detector.

\subsection{Structural determination and refinement}

The crystallographic data were processed with IMOSFLM [17] and scaled with SCALA from the CCP4 program suite [18]. Both structures of GCaMP6m monomer and dimer were determined by molecular replacement with Phaser [19] and the structure of the parent version GCaMP5G (PDB code: 3SG4) was used as a starting model. The structure was refined by using Phenix.refine [20,21] with manual modelling using COOT [22] between refinement cycles. The quality of the final models was checked with MolProbity [23]. All the figures were rendered in PyMOL (http:// www.pymol.org). The atomic coordinates of GCaMP6m monomer and dimer have been deposited in the Protein Data Bank with the accession codes 3WLD and 3WLC, respectively.

\section{Results and discussion}

\subsection{Overall structure of $\mathrm{Ca}^{2+}$-bound GCaMP6m}

The intact GCaMP contains an N-terminal RSET module preceding the M13 peptide, which functions to facilitate the expression and purification of recombinant GCaMP proteins $[12,13]$. Given that the RSET module has no effect on the $\mathrm{Ca}^{2+}$ sensing ability of GCaMP [12], we truncated the RSET of GCaMP6m and refer to this truncated form as GCaMP6m hereafter. Like other GCaMP variants, GCaMP6m, in the presence of $\mathrm{Ca}^{2+}$, existed in both a monomeric and a dimeric state during the purification process, which was successfully separated by gel filtration chromatography and crystallized for structure determination. 
The statistics for crystallographic data collection and structure refinement are summarized in Table 1. Structure of the dimeric GCaMP6m adopts a domain-swapped assembly, highly similar to the previously determined GCaMP2 dimer structure [12,13] (Figure 1). Formation of the dimer largely results from high protein concentration and crystallization condition; the dimer indeed has poor fluorescence properties that are insensitive to $\mathrm{Ca}^{2+}[12,13]$. Thus, we confined our in-depth structural analyses onto the functionally relevant GCaMP6m monomer.

Like all early versions of GCaMP, the overall structure of $\mathrm{Ca}^{2+}$-bound GCaMP6m could be divided into two global domains, the cpEGFP moiety and the $\mathrm{Ca}^{2+}$-induced CaMM13 peptide complex (Figure 2A). cpEGFP adopts a $\beta$ barrel fold with the chromophore, formed by ${ }_{223} \mathrm{TYG}_{225}$,

Table 1 Data collection and refinement statistics ${ }^{\text {a) }}$

\begin{tabular}{|c|c|c|}
\hline & GCaMP6m monomer & GCaMP6m dimer \\
\hline \multicolumn{3}{|l|}{ Data collection } \\
\hline Space group & $P 4{ }_{1}{ }_{1} 2$ & $C 2$ \\
\hline \multicolumn{3}{|l|}{ Cell dimensions } \\
\hline$a, b, c(\AA)$ & $a=b=120.88, c=97.62$ & $\begin{array}{l}a=124.44, b=46.86 \\
c=68.34\end{array}$ \\
\hline$\alpha, \beta, \gamma\left(^{\circ}\right)$ & $\alpha=\beta=\gamma=90.00$ & $\alpha=\gamma=90.00, \beta=99.36$ \\
\hline Wavelength $(\AA)$ & 1.5418 & 1.5418 \\
\hline Resolution ( $\mathrm{A})$ & $\begin{array}{l}60.44-2.70 \\
(2.85-2.70)\end{array}$ & $\begin{array}{l}20.08-2.49 \\
(2.62-2.49)\end{array}$ \\
\hline Unique reflections & $20011(2774)$ & $13660(1826)$ \\
\hline$R_{\text {merge }}(\%)$ & $8.7(37.7)$ & $7.8(25.1)$ \\
\hline Average $I / \sigma(I)$ & $12.7(3.3)$ & $12.3(4.6)$ \\
\hline Completeness (\%) & $98.1(95.3)$ & $98.6(91.4)$ \\
\hline Redundancy & $5.3(4.1)$ & $3.5(3.2)$ \\
\hline \multicolumn{3}{|l|}{ Refinement } \\
\hline Resolution ( $)$ & $\begin{array}{l}54.06-2.70 \\
(2.84-2.70)\end{array}$ & $\begin{array}{l}20.08-2.49 \\
(2.68-2.49)\end{array}$ \\
\hline No. reflections & 19975 (2705) & $13659(2563)$ \\
\hline$R_{\text {work }} / R_{\text {free }}(\%)$ & $\begin{array}{l}17.11 / 21.15 \\
(23.36 / 30.63)\end{array}$ & $\begin{array}{l}17.70 / 22.48 \\
(19.70 / 25.68)\end{array}$ \\
\hline \multicolumn{3}{|l|}{ No. atoms } \\
\hline Protein & 3111 & 3103 \\
\hline Water & 162 & 160 \\
\hline Chromophore & 22 & 22 \\
\hline Calcium ion & 4 & 4 \\
\hline \multicolumn{3}{|l|}{$B$-factors $\left(\AA^{2}\right)$} \\
\hline Protein & 32.75 & 20.00 \\
\hline Water & 27.71 & 20.44 \\
\hline Chromophore & 17.16 & 7.78 \\
\hline Calcium ion & 31.97 & 19.19 \\
\hline \multicolumn{3}{|l|}{ r.m.s. deviations } \\
\hline Bond lengths ( $\mathrm{A})$ & 0.006 & 0.004 \\
\hline Bond angles $\left(^{\circ}\right)$ & 1.085 & 0.748 \\
\hline Dihedral angles $\left({ }^{\circ}\right)$ & 14.602 & 15.893 \\
\hline Chirality $\left(^{\circ}\right)$ & 0.066 & 0.055 \\
\hline Planarity ( & 0.004 & 0.003 \\
\hline
\end{tabular}

\footnotetext{
a) Values in parentheses are for the highest-resolution shell.
}

sitting at the $\alpha$-helix along the central axis of the barrel. The phenolic group of the chromophore points to a $\beta$-barrel surface entrance generated by EGFP circular permutation (Figure 2B). The M13 peptide is folded into a straight $\alpha$ helix and inserted into a long central channel of $\mathrm{Ca}^{2+}$-bound CaM (Figure 2C). The tightly bound CaM-M13 complex is located at one side of cpEGFP, appropriately covering the surface entrance of cpEGFP with the chromophore partially exposed (Figure 2A). Extensive interactions were observed at the interface between the cpEGFP and the CaM-M13 complex, mainly involving $\alpha 4, \alpha 5$ and the connecting loop in the CaM moiety. These interactions are critical for the occlusion of chromophore exposure and the consequent fluorescence change in response to $\mathrm{Ca}^{2+}$. Notably, Arg376 from $\alpha 4$ in CaM protrudes towards the surface entrance of cpEGFP and stabilizes the deprotonated phenolic group of the chromophore through water-mediated hydrogen bonds (Figure 2D). Tyr380, derived from generation of the parent version GCaMP5G [15] and located at the end of $\alpha 4$, provides a large phenolic group that further blocks solvent access to the chromophore (Figure 2D).

\subsection{Critical mutations on GCaMP5G enhance $\mathrm{Ca}^{2+}$ sensitivity of GCaMP6}

GCaMP6 is generated by introducing three point mutations, Thr381Arg, Ser383Thr and Arg392Gly, into its parent version GCaMP5G. Arginine substitution of Thr381 at the cpEGFP-CaM interface below the surface entrance creates new strong interactions between cpEGFP and CaM. The positively charged side-chain of Arg381 of CaM stretches into a local negatively charged area in cpEGFP and is fixed by forming two hydrogen bonds with the side-chain of Glu299 and the main-chain of Gly87 in cpEGFP (Figure 3A). In addition, the Ser383Thr substitution, right adjacent to $\operatorname{Arg} 381$ in GCaMP6, results in introduction of a methyl group and generation of new hydrophobic interactions with the two non-polar residues Val88 and Leu90 in cpEGFP (Figure 3A). Thus, the Thr381Arg and Ser383Thr substitutions shall facilitate and further stabilize $\mathrm{Ca}^{2+}$ bindinginduced interactions between the CaM-M13 complex and the cpEGFP moiety, thereby providing a plausible structural explanation for the increased sensitivity and fluoresce intensity observed with GCaMP6. Moreover, the third Arg392Gly substitution is located nearby one of the $\mathrm{Ca}^{2+}$ binding sites of CaM in GCaMP6 (Figure 3B). Consistent with this structural observation, this substitution has been shown to promote the compaction process of $\mathrm{CaM}$ and increase the $\mathrm{Ca}^{2+}$ affinity in the GCaMP5 study [15]. The three substitutions exist in all three types of GCaMP6, and the resulting structural alteration and effects on $\mathrm{Ca}^{2+}$ induced conformational changes described above underlie the enhanced $\mathrm{Ca}^{2+}$ sensitivity of GCaMP6. 

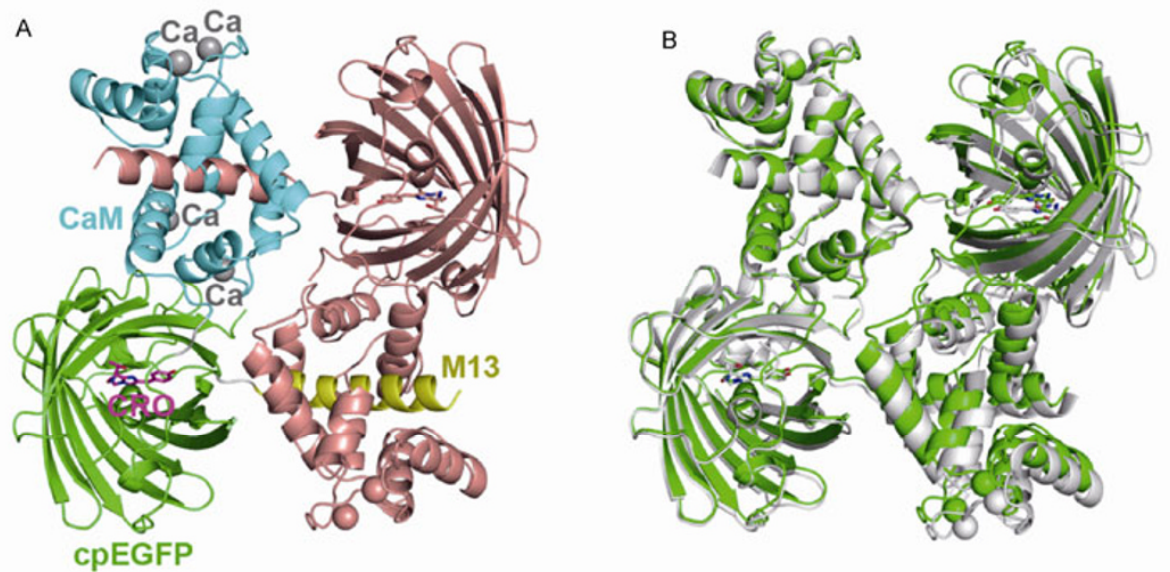

Figure 1 Structure and assembly of GCaMP6m dimer. A, Overall structure of GCaMP6m dimer in cartoon scheme. Of one GCaMP6m monomer in the dimer, M13, cpEGFP and CaM are colored in yellow, green and cyan, respectively; the chromophore (CRO) is shown as stick models and colored in magentas; the four $\mathrm{Ca}^{2+}$ bound to the CaM are shown as grey spheres. For clarity, the other GCaMP6m monomer in the dimer is totally colored in salmon. B, Structural superimposition of GCaMP6m dimer (in green) with GCaMP2 dimer (PDB code: 3EVU, in white).
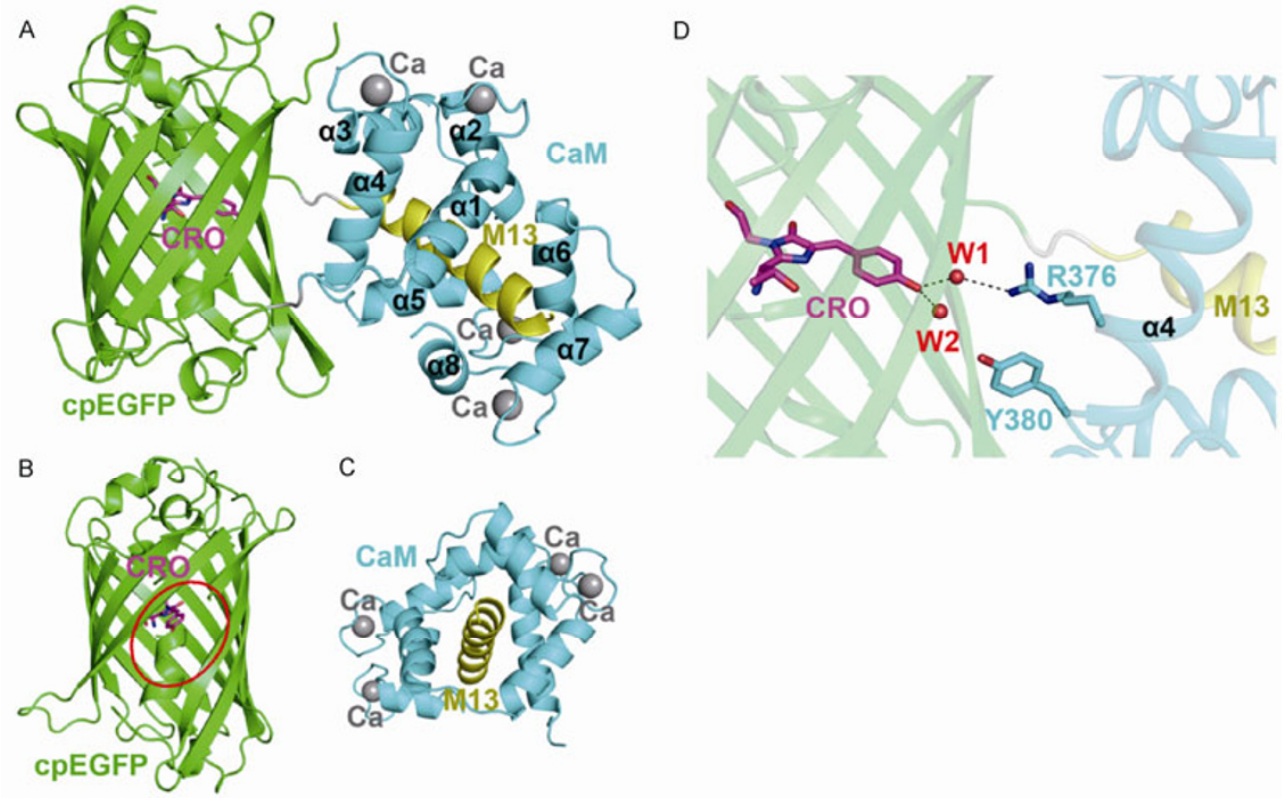

Figure 2 Structure of GCaMP6m monomer. A, Overall structure of GCaMP6m in cartoon scheme. GCaMP6m monomer is represented in the same scheme as that in Figure 1A. B, Side view of cpEGFP in GCaMP6m. The entrance on the surface of cpEGFP is highlighted in red circle. C, Top view of $\mathrm{Ca}^{2+}$-induced M13-CaM assembly. D, Close-up view of the chromophore stabilized and occluded by the CaM. The water molecules and residues of the CaM involved are shown as sphere and sticks, respectively. The hydrogen bonds are shown as black dashed lines.

\subsection{Additional substitutions in each type of GCaMP6 contribute to differential improvement}

In addition to the aforementioned three substitutions, each type of GCaMP6, compared with GCaMP5G, has additional substitutions to achieve their respective improved properties [16]. GCaMP6s, which shows the highest $\mathrm{Ca}^{2+}$ sensitivity but slow response kinetics, contains a Lys78His substitution in the cpEGFP moiety. According to the structure of GCaMP6m we determined, this substitution is expected to strengthen the hydrogen-bond network interactions among Glu60, Arg80 and Glu386 at the cpEGFP-CaM interface.
Such structural alteration shall contribute to the blocking of solvent access to the chromophore (Figure 4A). Thus, the structural effect of Lys78His mutation is consistent with its increased highest fluoresce intensity and relatively slow kinetics. GCaMP6f is characterized by its fastest $\mathrm{Ca}^{2+}$ sensing kinetics, compared with GCaMP6s and GCaMP6m. GCaMP6f contains the Ala317Glu substitution at the M13-CaM interface (rather than the cpEGFP-CaM interface). Ala317 is involved in the extensive hydrophobic interactions between CaM and M13, and introduction of a long charged side chain likely causes a destabilizing effect on the $\mathrm{Ca}^{2+}$ induced CaM-M13 assembly (Figure 4B), 


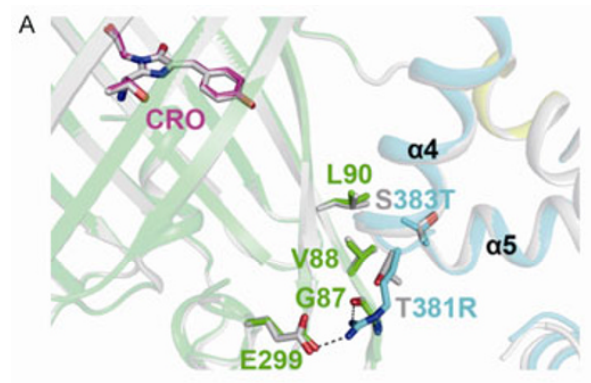

B

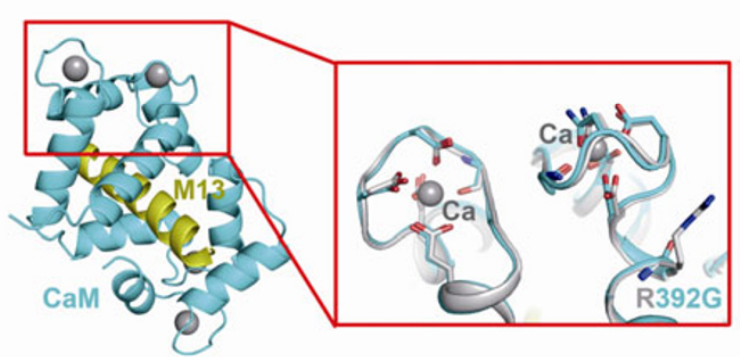

Figure 3 Critical mutations of GCaMP6 on GCaMP5G. A, Structural comparison of GCaMP6m an`d GCaMP5G (PDB code: 3SG4, light grey color) and close-up view of the T381R and S383T substitutions. B, Structural view of the R392G substitution nearby the $\mathrm{Ca}^{2+}$ binding sites in the CaM-M13 complex.

thereby accelerating the $\mathrm{Ca}^{2+}$ sensing kinetics. As for GCaMP6m, it possesses two unique substitutions, Met378-
Gly and Lys379Ser, located between the CaM and the cpEGFP. Compared with the crystal structure of GCaMP5G, both substitutions appear to play no direct and evident roles in either strengthening or weakening the interactions between CaM and cpEGFP (Figure 4C). However, these two residues are spatially close to interacting residues in the $\mathrm{CaM}$ and cpEGFP interface. Shortening of the side chains of the two residues and the resulting decreased entropy may reduce the structural unfitness and promote $\mathrm{Ca}^{2+}$-induced interaction between the CaM and cpEGFP interface. As a result, GCaMP6m shows a balanced performance between the fluorescent intensity and $\mathrm{Ca}^{2+}$ response kinetics [16].

In summary, we presented detailed structural analyses of GCaMP6 in comparison with its parent version GCaMP5G, and revealed the structural basis for the outperformance of this newly developed $\mathrm{Ca}^{2+}$ indicator. Three substitution mutations and the resulting changes of local structure and interaction explain the ultrasensitivity and increased fluorescence intensity common to all three versions of GCaMP6. Each particular substitution in the three GCaMP6 is also structurally consistent with their differential sensitivity and intensity, maximizing the potential of using GCaMP6 in solving diverse problems in neuronal research and calcium signaling. Our studies shall also be beneficial to further structure-guided optimization of the current GCaMP and facilitate the design of novel GECIs.
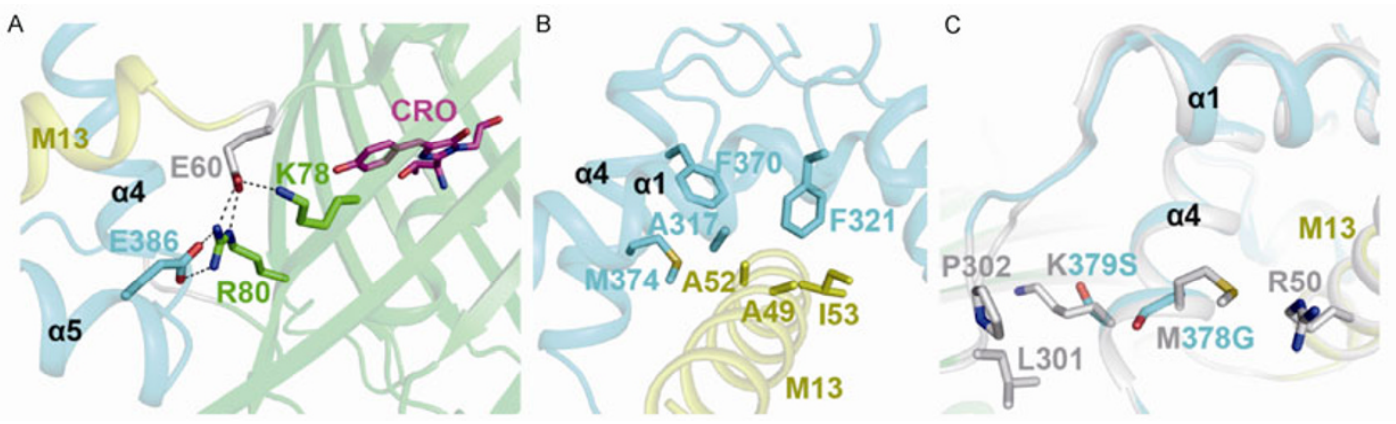

Figure 4 Additional substitutions in each type of GCaMP6. A, Close-up view of K78 of GCaMP6m and the related hydrogen-bond network at the CaM-cpEGFP interface. B, Close-up view of A317 of GCaMP6m and the related hydrophobic interactions at the M13-CaM interface. C, Structural comparison of GCaMP6m and GCaMP5G and close-up view of M378G/K379S substitutions and some neighboring residues. GCaMP5G is represented in the same scheme as that in Figure 3.

Crystallographic data were collected at Institute of Biophysics, Chinese Academy of Sciences and School of Life Sciences, Tsinghua University. This work was supported in part by an International Early Career Scientist grant from the Howard Hughes Medical Institute to Shao Feng, and grant from the National Basic Research Program of China (2011CB910304 and 2011CB911103) to Wang DaCheng and National Natural Science Foundation of China (31100535) to Ding JingJin.

1 Tank DW, Sugimori M, Connor JA, Llinás RR. Spatially resolved calcium dynamics of mammalian Purkinje cells in cerebellar slice. Science, 1988, 242: 773-777

2 Sabatini BL, Oertner TG, Svoboda K. The life cycle of $\mathrm{Ca}^{2+}$ ions in dendritic spines. Neuron, 2002, 33: 439-452
3 Kerr R, Lev-Ram V, Baird G, Vincent P, Tsien RY, Schafer WR. Optical imaging of calcium transients in neurons and pharyngeal muscle of C. elegans. Neuron, 2000, 26: 583-594

4 Ohki K, Chung S, Ch'ng YH, Kara P, Reid RC. Functional imaging with cellular resolution reveals precise micro-architecture in visual cortex. Nature, 2005, 433: 597-603

5 Jia H, Rochefort NL, Chen X, Konnerth A. Dendritic organization of sensory input to cortical neurons in vivo. Nature, 2010, 464: 1307-1312

6 Huber D, Gutnisky DA, Peron S, O'Connor DH, Wiegert JS, Tian L, Oertner TG, Looger LL, Svoboda K. Multiple dynamic representations in the motor cortex during sensorimotor learning. Nature, 2012, 484: 473-478

7 Stosiek C, Garaschuk O, Holthoff K, Konnerth A. In vivo two-photon 
calcium imaging of neuronal networks. Proc Natl Acad Sci USA, 2003, 100: 7319-7324

8 Tour O, Adams SR, Kerr RA, Meijer RM, Sejnowski TJ, Tsien RW, Tsien RY. Calcium Green FlAsH as a genetically targeted small-molecule calcium indicator. Nat Chem Biol, 2007, 3: 423-431

9 Palmer AE, Tsien RY. Measuring calcium signaling using genetically targetable fluorescent indicators. Nat Prot, 2006, 1: 1057-1065

10 Burgoyne RD. Neuronal calcium sensor proteins: generating diversity in neuronal $\mathrm{Ca}^{2+}$ signalling. Nat Rev Neurosci, 2007, 8: 182-193

11 Nakai J, Ohkura M, Imoto K. A high signal-to-noise $\mathrm{Ca}^{2+}$ probe composed of a single green fluorescent protein. Nat Biotechnol, 2001, 19: $137-141$

12 Wang Q, Shui B, Kotlikoff MI, Sondermann H. Structural basis for calcium sensing by GCaMP2. Structure, 2008, 16: 1817-1827

13 Akerboom J, Rivera JD, Guilbe MM, Malavé EC, Hernandez HH, Tian L, Hires SA, Marvin JS, Looger LL, Schreiter ER. Crystal structures of the GCaMP calcium sensor reveal the mechanism of fluorescence signal change and aid rational design. J Biol Chem, 2009, 284: 6455-6464

144 Tian L, Hires SA, Mao T, Huber D, Chiappe ME, Chalasani SH, Petreanu L, Akerboom J, McKinney SA, Schreiter ER, Bargmann CI, Jayaraman V, Svoboda K, Looger LL. Imaging neural activity in worms, flies and mice with improved GCaMP calcium indicators. Nat Methods, 2009, 6: 875-881

15 Akerboom J, Chen TW, Wardill TJ, Tian L, Marvin JS, Mutlu S, Calderón NC, Esposti F, Borghuis BG, Sun XR, Gordus A, Orger MB, Portugues R, Engert F, Macklin JJ, Filosa A, Aggarwal A, Kerr RA, Takagi R, Kracun S, Shigetomi E, Khakh BS, Baier H, Lagnado L, Wang SS, Bargmann CI, Kimmel BE, Jayaraman V, Svoboda K, Kim DS, Schreiter ER, Looger LL. Optimization of a GCaMP calcium indicator for neural activity imaging. J Neurosci, 2012, 32: 13819-13840

16 Chen TW, Wardill TJ, Sun Y, Pulver SR, Renninger SL, Baohan A,
Schreiter ER, Kerr RA, Orger MB, Jayaraman V, Looger LL, Svoboda K, Kim DS. Ultrasensitive fluorescent proteins for imaging neuronal activity. Nature, 2013, 499: 295-300

17 Battye TG, Kontogiannis L, Johnson O, Powell HR, Leslie AG. iMOSFLM: a new graphical interface for diffraction-image processing with MOSFLM. Acta Crystallogr D Biol Crystallogr, 2011, 67: 271-281

18 Winn MD, Ballard CC, Cowtan KD, Dodson EJ, Emsley P, Evans PR, Keegan RM, Krissinel EB, Leslie AG, McCoy A, McNicholas SJ, Murshudov GN, Pannu NS, Potterton EA, Powell HR, Read RJ, Vagin A, Wilson KS. Overview of the CCP4 suite and current developments. Acta Crystallogr D Biol Crystallogr, 2011, 67: 235-242

19 McCoy AJ, Grosse-Kunstleve RW, Storoni LC, Read RJ. Likelihoodenhanced fast translation functions. Acta Crystallogr D Biol Crystallogr, 2005, 61: 458-464

20 Afonine PV, Grosse-Kunstleve RW, Echols N, Headd JJ, Moriarty NW, Mustyakimov M, Terwilliger TC, Urzhumtsev A, Zwart PH, Adams PD. Towards automated crystallographic structure refinement with phenix.refine. Acta Crystallogr D Biol Crystallogr, 2012, 68: 352-367

21 Headd JJ, Echols N, Afonine PV, Grosse-Kunstleve RW, Chen VB, Moriarty NW, Richardson DC, Richardson JS, Adams PD. Use of knowledge-based restraints in phenix.refine to improve macromolecular refinement at low resolution. Acta Crystallogr D Biol Crystallogr, 2012, 68: 381-390

22 Emsley P, Lohkamp B, Scott WG, Cowtan K. Features and development of Coot. Acta Crystallogr D Biol Crystallogr, 2010, 66: 486-501

23 Chen VB, Arendall WB 3rd, Headd JJ, Keedy DA, Immormino RM, Kapral GJ, Murray LW, Richardson JS, Richardson DC. MolProbity: all-atom structure validation for macromolecular crystallography. Acta Crystallogr D Biol Crystallogr, 2010, 66: 12-21

Open Access This article is distributed under the terms of the Creative Commons Attribution License which permits any use, distribution, and reproduction in any medium, provided the original author(s) and source are credited. 